\title{
Endoscopic Management of Urethral Stricture Disease and Bladder Neck Contractures
}

\author{
James Furr, MD and Joel Gelman, MD
}

\begin{abstract}
This section aims to review general principles of endoscopic management of urethral stricture disease and posterior urethral stenosis, with a special focus on the indications for treatment and technical aspects of urethral dilation and internal incision.
\end{abstract}

Keywords: urethral stricture disease, bladder neck contractures, urethral dilation, direct vision internal urethrotomy

Urethral Stricture Disease

Etiology

A NTERIOR URETHRAL STRICTURE disease refers to a severe scarring process that occurs in the corpus spongiosum (spongiofibrosis) and ultimately results in narrowing of the urethra. As the lumen reduces from its normal caliber $(\sim 30 \mathrm{~F}$ except in the fossa navicularis where the normal caliber is 22F-24F), voiding dysfunction and obstructive symptoms will occur. Symptoms become more common as the caliber decreases to less than $16 \mathrm{~F}$, and most strictures are less than $10 \mathrm{~F}$ to $12 \mathrm{~F}$ in caliber. More severe cases may present with complete retention, bladder stones, or recurrent urinary tract infections (UTIs). ${ }^{1,2}$

Historically, inflammation and untreated sexually transmitted gonococcal infections were considered a primary cause of strictures. In contemporary series, however, common causes include straddle trauma, pelvic fracture urethral injury, instrumentation, prior hypospadias surgery, and prior prostate surgery, and/or radiation. ${ }^{3}$ Inflammatory strictures are commonly associated with lichen sclerosis also known as balanitis xerotica obliterans, which can be associated with panurethral stricture disease, which is generally not responsive to endoscopic management. Overall, urethral stricture disease is common, with a reported prevalence as high as 600 per $100,000 .^{4}$

\section{Patient assessment and indication for intervention}

Index of suspicion for urethral stricture disease should be high in patients with obstructive voiding symptoms especially younger patients and older men who do not respond to alpha blocker medication, a history of trauma, transurethral surgery, prior prostate cancer treatment, prior hypospadias repair, and lichen sclerosis. The America Urological Association (AUA) released updated guidelines in 2016, which gave guidance with regard to initial workup. Guidelines recommend validated symptom score, such as AUA-Symptom Index as well as uroflowmetry in the initial assessment of patients suspected of having urethral stricture disease. However, ultimate diagnosis requires imaging and endoscopic evaluation. ${ }^{5}$

A thorough anatomic assessment with a retrograde urethrogram and voiding cystourethrogram is required to identify the location and length of the stricture. We have observed that the vast majority of our patients who presented with a history of prior endoscopic treatment never had imaging or were informed urethroplasty was a treatment option before undergoing endoscopic treatment with dilation or direct vision internal urethrotomy (DVIU). This was also true for repeat treatments in the majority of patients. ${ }^{6}$ This was unfortunate as without imaging subsequent to a stricture diagnosis by urethroscopy, one cannot accurately assess stricture length, and both longer and recurrent strictures are generally managed but not cured with endoscopic treatment, whereas urethroplasty can offer cure rates up to over $98 \% .^{7-9}$ Given these observations, guidelines are now published and indicate that endoscopic treatment should generally be reserved for short bulbar strictures, and recurrent or longer strictures are best managed by urethroplasty. Principles of urethral imaging technique are reviewed in another section and are beyond the scope of this specific review.

\section{Endoscopic techniques}

Preoperative planning. Preoperatively, the patient's urine should be evaluated by urinalysis with microscopy, and

Department of Urology, University of California, Irvine, Orange, California, USA.

(C) James Furr and Joel Gelman 2020; Published by Mary Ann Liebert, Inc. This Open Access article is distributed under the terms of the Creative Commons Attribution Noncommercial License (http://creativecommons.org/licenses/by-nc/4.0/) which permits any noncommercial use, distribution, and reproduction in any medium, provided the original author(s) and the source are cited. 
sent for culture if pyuria and/or bacteriuria is seen. Any infection should be treated with culture-specific antibiotics. A single dose of perioperative intravenous antibiotics should be given based on AUA recommendations. ${ }^{10}$ In the emergent setting, some endoscopic procedures such as dilation can be performed at bedside with local, intraurethral anesthetic. However, the most ideal setting would involve general anesthesia or at the very least spinal or epidural anesthesia.

Patient positioning. For endoscopic, transurethral management of urethral stricture disease, patients are typically placed in the dorsal lithotomy position. Following administration of anesthesia, the patient's buttocks are moved to the edge of the bed. The lower legs are placed in stirrups. The arms are either tucked to the sides or secured in arm boards. When performing DVIU the dorsal lithotomy position allows for the best mobility of the rigid cystoscopic instruments within the urethra. Urethral dilation could be performed in the supine position with certain methods, but lithotomy best allows all options. In emergent circumstances, blind passage of dilation instruments could be performed in the supine position. However, this is not recommended within the controlled, operating room setting, and with newer instrumentation, blind dilation can be avoided in all cases.

Urethral dilation: surgical steps. Urethral dilation has historically been a well-characterized technique to the management of urethral stricture disease. Dilation is typically fast, offers immediate results, and is technically straightforward. The concept behind dilation involves the sequential stretching, not tearing of the stricture.

When severe narrowing of the urethral meatus or fossa navicularis is noted, dilation can offer an alternative treatment to meatotomy or flap reconstruction. Another potential need for meatal dilation is the inability to pass a resectoscope. The standard urethral meatus ranges from $22 \mathrm{~F}$ to $24 \mathrm{~F}$, so minimal dilation may be required to accommodate a $26 \mathrm{~F}$ resectoscope. We recommend the use of McCrea sounds (Fig. 1) as the angle is less severe than Van Buren sounds (Fig. 2) decreasing the risk of false passage.

When the stricture is located in the bulbar urethra, we recommend against the use of blind passage of Van Buren or McCrea sounds, as fossa navicularis strictures can occur

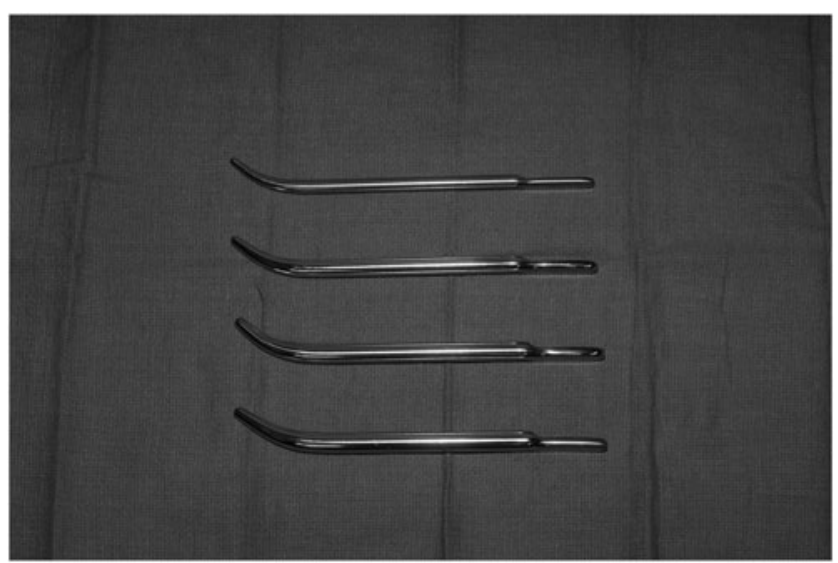

FIG. 1. Image of McCrea sounds.

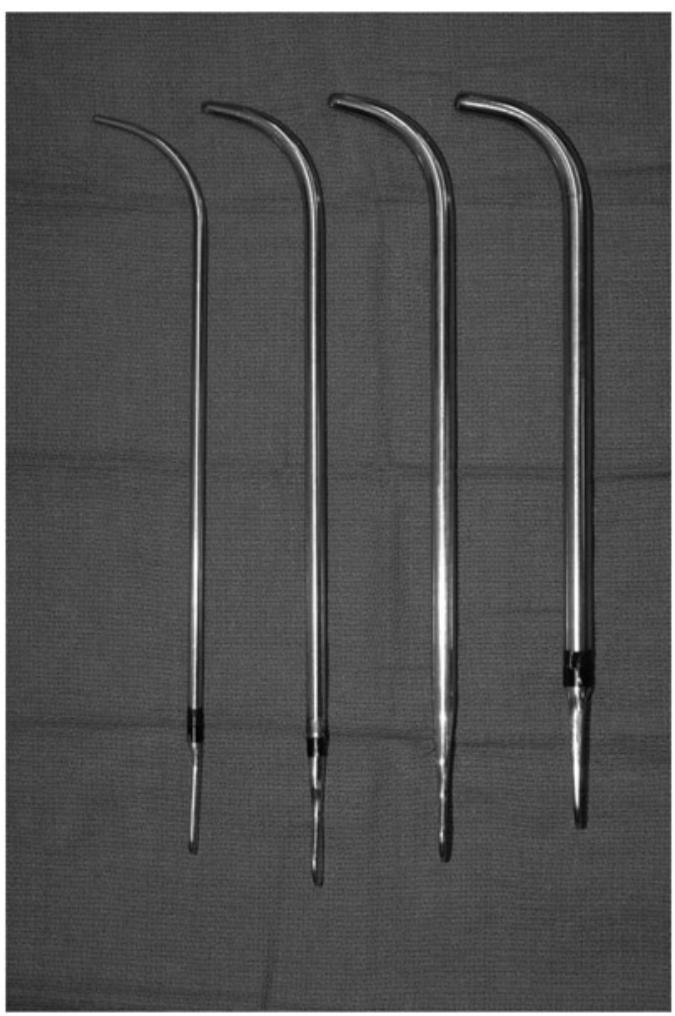

FIG. 2. Image of Van Buren sounds.

since the distal penile urethra will be dilated more than desired to achieve the appropriate luminal diameter of the bulbar urethra. Instead, filiforms or followers can be used without traumatizing the fossa navicularis (Fig. 3). Placement of guidewire is typically performed under direct visualization with either a $22 \mathrm{~F}$ rigid cystoscope or a flexible cystoscope (Fig. 4). Once access to the bladder is confirmed, a variety of instruments can be used to perform the sequential dilations. Commonly, Heyman followers are used to pass over the guidewire until the set French $(\sim 24 \mathrm{~F}-28 \mathrm{~F})$ is reached.

Alternatively, the use of balloon dilation within the urethra has been described using either Uromax Ultra ${ }^{\mathrm{TM}}$ (Boston Scientific) or Cook Urologic Accent Ureteral Dilation

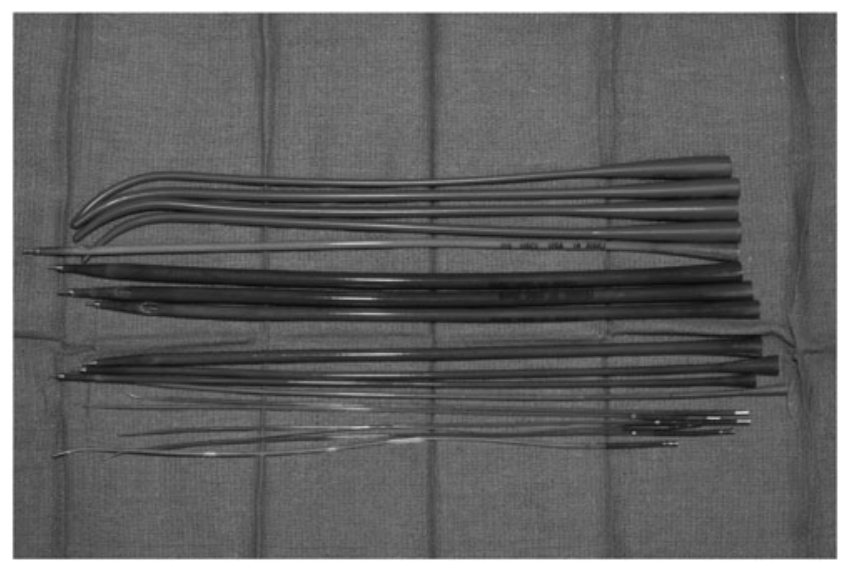

FIG. 3. Filiform followers and Heyman dilators often used with the blind dilation technique. 


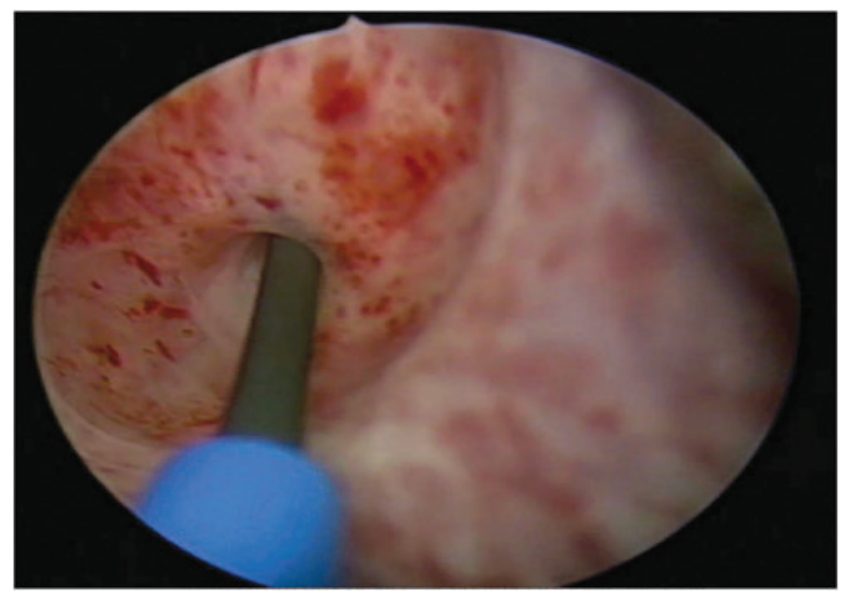

FIG. 4. Placement of guidewire under direct visualization past the level of the stricture into the bladder.

Balloon Catheter. The Uromax Ultra is available in two sizes, $15 \mathrm{~F}$ and $24 \mathrm{~F}$. The advantage of the $15 \mathrm{~F}$ is that it can be passed through a flexible scope, while all others cannot. We typically use the Cook Ureteral Dilator, which is $5 \mathrm{~F}, 80-\mathrm{cm}$ open lumen catheter with $4-\mathrm{cm}$ long, $30 \mathrm{~F}$ balloon. ${ }^{11}$ With this technique, the balloon dilator is passed over a hydrophilic guidewire and used to dilate the stricture for 5 to 10 minutes to $\sim 10$ to 20 atmosphere (atm). ${ }^{11,12}$ Since the dilator is passed through the rigid cystoscope, the dilation can be performed under direct visualization (Figs. 5 and 6). As this procedure is performed under direct visualization, it is more controlled than the blind passage of filiforms or Heyman followers. At the conclusion of the procedure, the catheter should be passed over indwelling guidewire to ensure no false passage creation in the traumatized urethral mucosa.

DVIU surgical steps. DVIU presents a very controlled alternative to urethral dilation. The incision is made under direct visualization, and can be performed using a number of different modalities such as cold knife or holmium laser technology.

The patient is placed in the dorsal lithotomy position with stirrups with chlorhexidine or betadine surgical preparation.

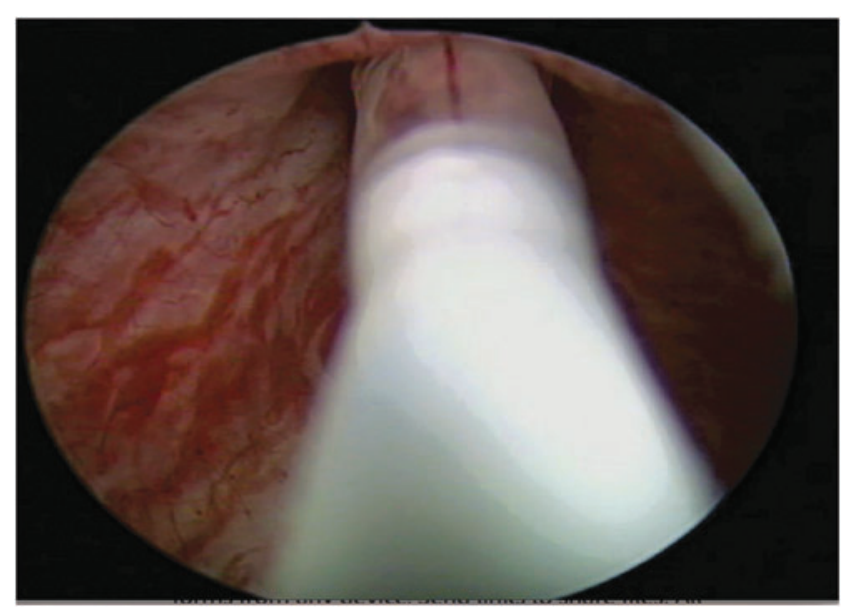

FIG. 5. Passage of Cook Dilator over the wire across the stricture.

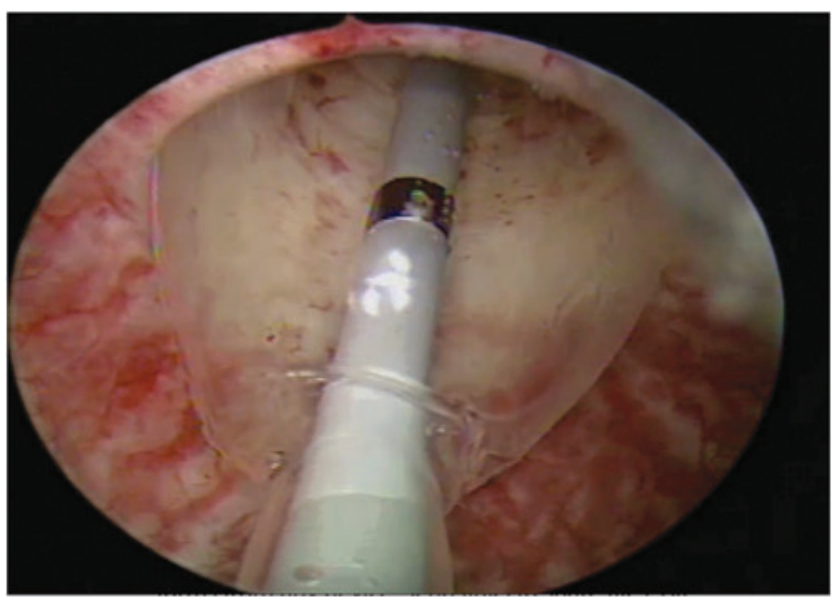

FIG. 6. Gentle balloon dilation of stricture under direct visualization.

A $22 \mathrm{~F}$ rigid cystoscope is used to visualize the stricture. No attempt should be made to traverse the stricture with the scope as this could traumatize healthy urothelium and produces dilation effect. Under direct visualization, a guidewire is passed in a retrograde fashion into the bladder. Passage of guidewire is a critical portion of the procedure as it allows for safety access to the bladder in the event of significant bleeding or trauma, which can make retrograde passage of Foley catheter difficult.

When the urethrotomy is performed with a cold knife, an urethrotome is used instead of the cystoscope. The majority of urethrotomes have an obturator and a $21 \mathrm{~F}$ irrigation sheath, which can be blindly placed to the level of the stricture (Fig. 7). Once the sheath is in an appropriate position, the urethrotome in advanced with a zero-degree lens and the cold knife at the 12 o'clock position. Upward pressure is applied through the stricture. The 12 o'clock position is typically the best to avoid the vasculature at the 3 and 9 o'clock positions in the bulbar urethra. The incision is repeated until a fullthickness defect is made in the stricture and the spongiosum is encountered. Once the appropriate luminal diameter is reached, the urethrotome should be advanced to evaluate the urethra and bladder proximal to the stricture. As opposed to the traditional deep incisions at the 12 o'clock position, our

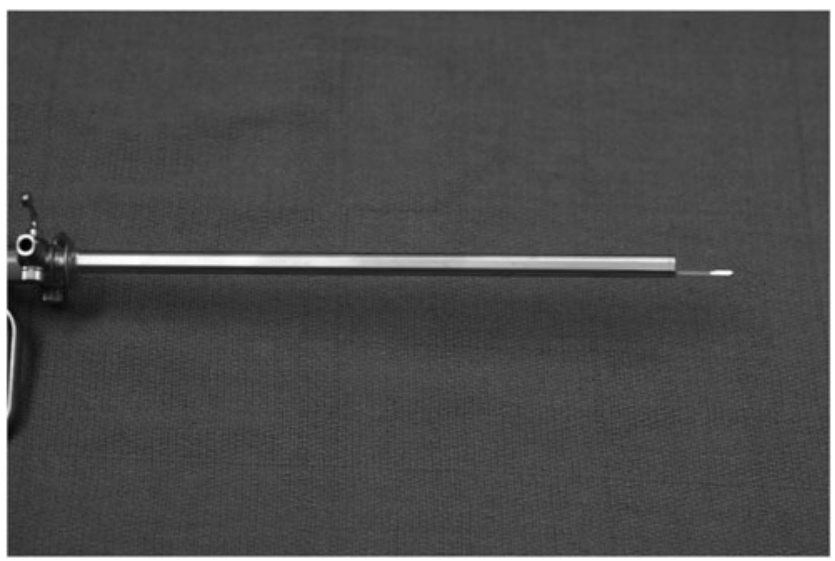

FIG. 7. Commonly used urethrotome with blade in place. 
preference is to perform three to four radial incisions that are not full thickness (Fig. 8).

If holmium laser is utilized to incise the stricture, the entire procedure can be performed with the $22 \mathrm{~F}$ rigid cystoscope. Similar to the technique utilized for cold knife, a guidewire is passed to gain access to the bladder. The cystoscope is removed and passed alongside the indwelling guidewire. A 365 micron laser is utilized for the incision and passed through the working port of the cystoscope. Either the 12 o'clock position of the urethral stricture or three to four radial incisions can be incised. The range of setting that can be employed for the holmium laser are $0.4 \mathrm{~J}$ to $1.4 \mathrm{~J}$ and $6 \mathrm{~W}$ to $12 \mathrm{~W}$, although the literature does not support any specific setting.

Once the stricture is incised and able to accommodate the cystoscope, a final $18 \mathrm{~F}$ to $20 \mathrm{~F}$ Councill tip Foley catheter should be placed over the indwelling guidewire. The catheter should then be irrigated to ensure placement and hemostasis.

\section{Postoperative care}

The patient is typically discharged from outpatient surgery with the indwelling Foley in place. There is no definitive consensus regarding the efficacy of leaving a catheter in place after dilation or urethrotomy. ${ }^{13}$ The authors of this review typically leave an indwelling catheter in place for $\sim 3$ days after intervention. Extended catheterization time may result in increased inflammation and subsequent fibrosis. Furthermore, no consensus exists with regard to intermitted catheterization following urethrotomy or dilation. Some retrospective analyses report modest improvement in stricture recurrence rate with intermittent catheterization. No level I evidence exists, although many groups employ weekly to biweekly with a $14 \mathrm{~F}$ catheter. ${ }^{14-16}$

\section{Complications and intraoperative troubleshooting}

Overall complication rate of endoscopic management of stricture disease is reported to be as high as $6.5 \%$. Immediate

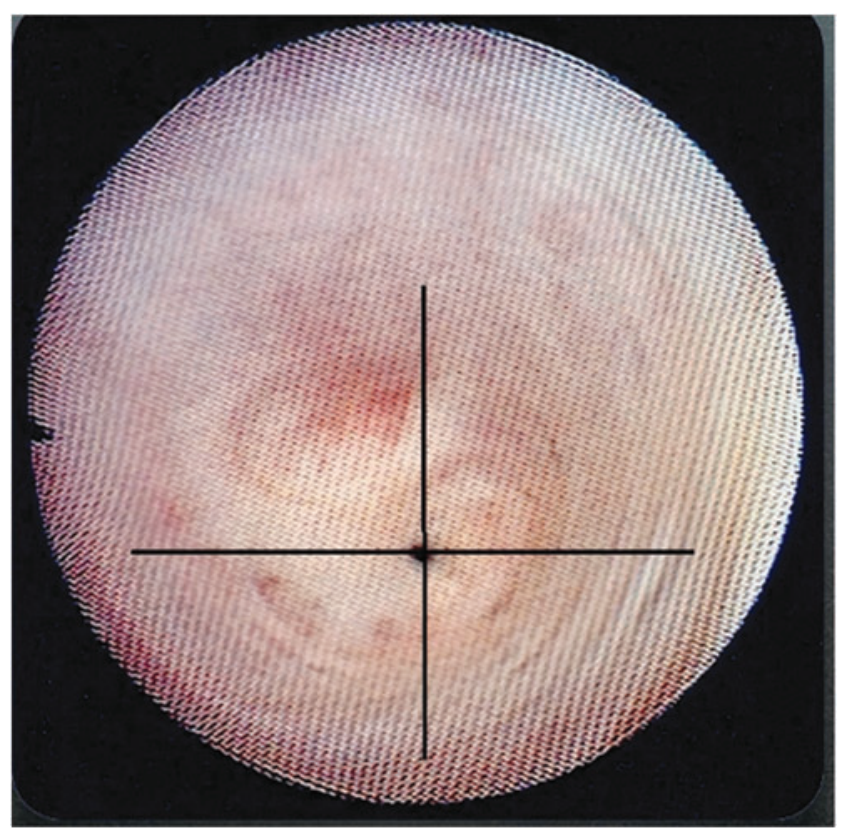

FIG. 8. Options for incision location for direct vision internal urethrotomy. complications include significant gross hematuria $(2 \%)$ and UTI. ${ }^{1,17}$ Under most circumstances the hematuria will resolve with large-bore $(22 \mathrm{~F}-24 \mathrm{~F})$ catheterization and bladder irrigation. Some degrees of erectile dysfunction and urinary incontinence have been reported to be as high as $5 \%$ and $4 \%$, respectively. ${ }^{17}$ Patient habitus and comorbid status rarely result in increased complexity. However, patients should be counseled on suprapubic catheter placement and antegrade access in the event that retrograde access cannot be obtained.

\section{Outcomes}

There does not appear to be a difference in efficacy between cold knife or laser technique, however, some metanalysis has reported twice as high a complication rate $(6 \% \mathrm{vs}$ $12.5 \%){ }^{17,18}$ Historically, short follow-up studies have reported stricture-free rate nearing $80 \% .{ }^{1}$ However, recent reports with long-term follow-up have showed that this success is not durable, and success rates are as low as $8 \% .{ }^{19}$ For complex, long $(>2 \mathrm{~cm})$ or recurrent strictures, the long-term success rates are likely much worse. Despite the poor durability of urethrotomy and dilation, there remains a roll for endoscopic management, especially in patient with short, simple stricture or patients with comorbid status precluding them from major reconstruction. The overall success rate is difficult to accurately report. There is significant heterogeneity in stricture length, follow-up time, and definition of success across the literature, with states' success rates ranging from $8 \%$ to $80 \%$ in some series. ${ }^{20-23}$

\section{Bladder neck contractures and posterior urethral stenosis}

Etiology. Bladder neck contracture (BNC) and posterior urethral stenosis is a relatively common occurrence following prostatic surgery for both benign and malignant prostatic conditions. Incidence following radical prostatectomy (either through open or robotic approach) can reach as high as $17.5 \%$ and as high as $12 \%$ for Transurethral Resection of Prostate (TURP). ${ }^{24}$ Furthermore, radiation effects and trauma can result in stenosis at the membranous or prostatic urethra. Additional risk factors include anastamotic tension, hematoma, or urine leak. ${ }^{25,26}$

\section{Patient assessment and indication for intervention}

Similar to anterior urethral stricture disease, patient will often present with obstructive voiding, dysuria, recurrent UTIs, or bladder stones. Index of suspicion for BNC rises in patients with the known risk factors, such as prior TURP, radical prostatectomy, brachytherapy, or radiation. Proper assessment involves detailed history, especially past surgical history. Furthermore, endoscopic assessment with proper documentation of location and diameter of the stenosis is required before any intervention. Similar to urethral stricture disease, radiologic assessment involves retrograde urethrogram and voiding cystourethrogram (if possible). Inability to bypass the stenosis with rigid or flexible cystoscope in patients with known voiding symptoms is an indication for intervention. While open reconstruction of the bladder neck has been described, the majority of patients are still managed with endoscopic techniques. 


\section{Endoscopic techniques}

Preoperative planning and patient positioning. Preoperative management for intervention for BNC is similar to that of other transurethral procedures for anterior urethral strictures or prostatic procedures. Preoperative urine cultures are obtained and treated before intervention. If urine cultures are negative, then intravenous antibiotics are given based on AUA guidelines. The patient is then placed in dorsal lithotomy position as outlined in prior sections.

Transurethral incision of BNC-surgical steps. A variety of instruments can be used to incise the $\mathrm{BNC}$, ranging from loop resection, cold knife incision, Collings knife incision, or laser. Regardless of the source of energy or incising instrument used, the principle remains consistent-to incise the bladder neck to a luminal diameter that can freely accommodate the rigid scope.

To start the procedure, the full extent of the urethra is examined with a 22F cystoscope. A 0.035 in Sensor PTFENitinol guidewire (Boston Scientific) is passed directly into the bladder under direct visualization. Most frequently we utilize a Collings knife, which is assembled within a $24 \mathrm{~F}$ or $26 \mathrm{~F}$ continuous flow resectoscope and initially passed into the urethra through a visual obturator. With the guidewire visible, the bladder neck is incised on monopolar cut setting at $50 \mathrm{~V}$. The incisions are made at the 3 and 9 o'clock positions until perivesicular fat is encountered. The resectoscope should be able to be passed freely into the bladder if deep enough incisions have been made. Alternatively, a cold knife can be used through a $21 \mathrm{~F}$ urethrotome. Tri or quadrant incisions can be made in a radial fashion until perivesicular fat is reached. To conclude, hemostasis is achieved and a $20 \mathrm{~F}$ to $22 \mathrm{~F}$ Councill tip catheter is passed over the guidewire into the bladder.

Balloon dilation of bladder neck contraction-surgical steps. Some groups have promoted the use of balloon dilation at the level of the bladder neck. Similar to balloon dilation within the anterior urethra, a 24F Uromax Ultra (Boston Scientific) balloon dilator set has been described in the literature. Initially, a $22 \mathrm{~F}$ cystoscope is placed into the urethra and the bladder is accessed with a guidewire. The balloon dilator is passed over the guidewire into the bladder so that the balloon traverses the contracture. Another, flexible cystoscope or $17 \mathrm{~F}$ cystoscope can be passed alongside the balloon device so that the dilation can occur under direct visualization. The contracture is then dilated to a level of 15 atm for 3 to 5 minutes. Some series have reported combining balloon dilation with incision, which has yielded positive results. $^{27}$

\section{Postoperative care}

At the conclusion of the procedure, regardless of method, a $20 \mathrm{~F}$ to $22 \mathrm{~F}$ urethral catheter is left for 3 to 5 days. The patient is assessed at $\sim 3$ months with cystoscopy, uroflow, and postvoid residual. Close followup is typically maintained at least every 6 months to ensure a durable response. A percentage of patients will also have incontinence, and will need at least another cystoscopic evaluation at 3 months to ensure patency before placement of an artificial urinary sphincter. ${ }^{27}$

\section{Outcomes and complications}

A durable response is frequently achieved with the aforementioned conservative, endoscopic techniques. Acceptable success rates with modest follow-up are seen from $50 \%$ to $72 \%$ of the time. ${ }^{26,27}$ This is fortunate, especially given that recurrent failures may require open reconstruction with all associated complexity and morbidity. As with any transurethral procedure, UTI and hematuria can occur, but are typically easily managed with catheterization and antibiotics. Complications specific to this procedure include de novo stress incontinence. Rates of de novo stress incontinence vary greatly across the literature, although in our experience, the majority of men present with concurrent stress incontinence and BNC. ${ }^{28,29}$

\section{Troubleshooting: Management of Recurrence}

Despite the surgeon's best efforts, stricture recurrence is commonplace when managing strictures through endoscopic approaches. Transitioning to major reconstruction or repeated endoscopic interventions were historically felt to be the only options; however, recent work investigating scar modulation and antiproliferative injects have studied some modest success. Vanni and colleagues has shown increased success in injecting mitomycin $\mathrm{C}$ into recalcitrant BNC's following incision, although Redshaw and colleagues showed less successful outcomes with nearly a $7 \%$ rate of serious adverse events. ${ }^{30,31}$ Other scar modulating techniques that are being studied are injections of botulinum toxin type A, as well as system docetaxel and rapamycin. However, these agents remain in investigation phase and do not have robust cohorts or lengthy follow-ups. ${ }^{32}$

\section{Recommended Videos from Videourology}

1. Videourology 2018, Vol. 32, No. 2

Direct Visual Internal Urethrotomy Followed by Immediate Injection of Mitomycin C: A Safe and Feasible Procedure for Bulbar and Bulbomembranous Urethral Strictures

Wei Phin Tan, M. Ryan Farrell, and Laurence A. Levine https://www.liebertpub.com/doi/full/10.1089/vid.2017.0049

2. Videourology 2020, Vol. 34, No. 2

Robot-Assisted Laparoscopic Posterior Urethroplasty Using the da Vinci Single Port Robot

Min Suk Jun, Wen Liu, Geolani W. Dy, Xiaosong Meng, and Lee C. Zhao,

https://www.liebertpub.com/doi/full/10.1089/vid.2019.0095

\section{Author Disclosure Statement}

No competing financial interests exist.

\section{Funding Information}

No funding was received for this article.

\section{Supplementary Data}

Supplementary Video S1 


\section{References}

1. Hampson LA, McAninch JW, Breyer B. Male urethral strictures and their management. Nat Rev Urol 2014;11: 43-50.

2. Wong SS, Aboumarzouk OM, Narahari R, O'Riordan A, Pickard R. Simple urethral dilatation, endoscopic urethrotomy, and urethroplasty for urethral stricture disease in adult men. Cochrane Database Syst Rev 2012;12:CD006934.

3. Lumen N, Hoebeke P, Willemsen P, De Troyer B, Pieters R, Oosterlinck W. Etiology of urethral stricture disease in the 21st century. J Urol 2009;182:983-987.

4. Santucci RA, Joyce GF, Wise M. Male urethral stricture disease. J Urol 2007;177:1667-1674.

5. Wessells H, Angermeier KW, Elliott S, et al. Male urethral stricture: American Urological Association Guideline. J Urol 2017;197:182-190.

6. Wisenbaugh ES, De Grado JJ, Quinn R, Gelman J. Men with anterior urethral strictures are often treated without imaging or discussion of all treatment options. Urol Pract 2017;5:52-56.

7. Eltahawy EA, Virasoro R, Schlossberg SM, McCammon $\mathrm{KA}$, Jordan GH. Long-term followup for excision and primary anastomosis for anterior urethral strictures. J Urol 2007;177:1803-1806.

8. Santucci RA, Mario LA, McAninch JW. Anastomotic urethroplasty for bulbar urethral stricture: Analysis of 168 patients. J Urol 2002;167:1715-1719.

9. Virasoro R, Eltahawy EA, Jordan GH. Long-term followup for reconstruction of strictures of the fossa navicularis with a single technique. BJU Int 2007;100:1143-1145.

10. American Urological Association. Best practice policy statement on urologic surgery antimicrobial prophylaxis. American Urological Association Education and Research, Inc., 2008, p. 48 [Updated 2014 Jan]. https://www.auanet .org/guidelines/antimicrobial-prophylaxis-best-practicestatement

11. Gelman J, Liss MA, Cinman NM. Direct vision balloon dilation for the management of urethral strictures. J Endourol 2011;25:1249-1251; discussion 1251.

12. Yu SC, Wu HY, Wang W, et al. High-pressure balloon dilation for male anterior urethral stricture: Single-center experience. J Zhejiang Univ Sci B 2016;17:722-727.

13. Naude AM, Heyns CF. What is the place of internal urethrotomy in the treatment of urethral stricture disease? Nat Clin Pract Urol 2005;2:538-545.

14. Lauritzen M, Greis G, Sandberg A, Wedren H, Ojdeby G, Henningsohn L. Intermittent self-dilatation after internal urethrotomy for primary urethral strictures: A case-control study. Scand J Urol Nephrol 2009;43:220-225.

15. Bodker A, Ostri P, Rye-Andersen J, Edvardsen L, Struckmann J. Treatment of recurrent urethral stricture by internal urethrotomy and intermittent self-catheterization: A controlled study of a new therapy. J Urol 1992;148: 308-310.

16. Matanhelia SS, Salaman R, John A, Matthews PN. A prospective randomized study of self-dilatation in the management of urethral strictures. J R Coll Surg Edinb 1995; 40:295-297.

17. Jin T, Li H, Jiang LH, Wang L, Wang KJ. Safety and efficacy of laser and cold knife urethrotomy for urethral stricture. Chin Med J (Engl) 2010;123:1589-1595.

18. Wang L, Wang Z, Yang B, Yang Q, Sun Y. Thulium laser urethrotomy for urethral stricture: A preliminary report. Lasers Surg Med 2010;42:620-623.
19. Al Taweel W, Seyam R. Visual internal urethrotomy for adult male urethral stricture has poor long-term results. Adv Urol 2015;2015:656459.

20. Santucci R, Eisenberg L. Urethrotomy has a much lower success rate than previously reported. J Urol 2010;183: 1859-1862.

21. Mazdak H, Izadpanahi MH, Ghalamkari A, et al. Internal urethrotomy and intraurethral submucosal injection of triamcinolone in short bulbar urethral strictures. Int Urol Nephrol 2010;42:565-568.

22. Chung JH, Kang DH, Choi HY, et al. The effects of hyaluronic acid and carboxymethylcellulose in preventing recurrence of urethral stricture after endoscopic internal urethrotomy: A multicenter, randomized controlled, singleblinded study. J Endourol 2013;27:756-762.

23. Pal DK, Kumar S, Ghosh B. Direct visual internal urethrotomy: Is it a durable treatment option? Urol Ann 2017;9:18.

24. Ramirez D, Zhao LC, Bagrodia A, Scott JF, Hudak SJ, Morey AF. Deep lateral transurethral incisions for recurrent bladder neck contracture: Promising 5-year experience using a standardized approach. Urology 2013;82:1430-1435.

25. Simhan J, Ramirez D, Hudak SJ, Morey AF. Bladder neck contracture. Transl Androl Urol 2014;3:214-220.

26. Cotta BH, Buckley JC. Endoscopic treatment of urethral stenosis. Urol Clin North Am 2017;44:19-25.

27. Ramirez D, Simhan J, Hudak SJ, Morey AF. Standardized approach for the treatment of refractory bladder neck contractures. Urol Clin North Am 2013;40:371-380.

28. Anger JT, Raj GV, Delvecchio FC, Webster GD. Anastomotic contracture and incontinence after radical prostatectomy: A graded approach to management. J Urol 2005; 173 : 1143-1146.

29. Yurkanin JP, Dalkin BL, Cui H. Evaluation of cold knife urethrotomy for the treatment of anastomotic stricture after radical retropubic prostatectomy. J Urol 2001;165:1545-1548.

30. Vanni AJ, Zinman LN, Buckley JC. Radial urethrotomy and intralesional mitomycin $\mathrm{C}$ for the management of recurrent bladder neck contractures. J Urol 2011;186:156-160.

31. Redshaw JD, Broghammer JA, Smith TG, 3rd, et al. Intralesional injection of mitomycin $\mathrm{C}$ at transurethral incision of bladder neck contracture may offer limited benefit: TURNS Study Group. J Urol 2015;193:587-592.

32. Gallegos MA, Santucci RA. Advances in urethral stricture management. F1000Res 2016;5:2913.

\section{Address correspondence to: Joel Gelman, MD Department of Urology University of California Irvine 333 City Blvd. W \#2100 Orange, CA 92868 USA}

E-mail: jgelman@uci.edu
Abbreviations and Acronyms
Atm $=$ atmospheres
AUA $=$ American Urological Association
$\mathrm{BNC}=$ bladder neck contracture
$\mathrm{DVIU}=$ direct vision internal urethrotomy
TURP $=$ Transurethral Resection of Prostate
$\mathrm{UTI}=$ urinary tract infection 